BULLETIN OF THE

AMERICAN MATHEMATICAL SOCIETY

Volume 77, Number 1, January 1971

\title{
BOUNDARY VALUES OF BOUNDED HOLOMORPHIC FUNCTIONS OF SEVERAL VARIABLES
}

\author{
BY HAROLD S. SHAPIRO
}

Communicated by F. W. Gehring, July 14, 1970

1. Introduction. In this note we state some results concerning the structure of a function which is the Fatou limit, on the distinguished boundary, of a bounded holomorphic function in a product of halfplanes. Such a function is, equivalently, an element of $L^{\infty}\left(R^{n}\right)$ whose (distributional) Fourier transform is supported in the "first quadrant", i.e. the set

$$
Q^{+}=\left\{x: x_{1} \geqq 0, \cdots, x_{n} \geqq 0\right\} .
$$

A typical result (consequence of Theorem 1) is that if such a function tends to a limit $\lambda$ as $x \rightarrow x^{0}$ from inside an open cone with vertex at $x^{0}$, then it tends "on the average" to $\lambda$ as $x \rightarrow x^{0}$ from inside any open cone with vertex at $x^{0}$. In particular, if such a function tends to limits in each of two open cones with a common vertex, these limits must be equal; for $n=1$ (when the cones are half-lines), this is a classical theorem of Pringsheim and Lindelöf. Theorem 2 is a Tauberian theorem for $H^{\infty}$ boundary values; specialized to one variable it yields (among other things) a new proof of Lindelöf's theorem that a bounded analytic function which tends to a limit radially does so also within an angle, as well as an apparently new relation between the average behaviour of an $H^{\infty}$ boundary function to the left, and that to the right, of a given point. Theorem 3 shows that a much stronger localization of uniform convergence for, say, Fejér means, is valid for $H^{\infty}$ boundary functions than for bounded measurable functions generally; for example, if the restriction of an $H^{\infty}$ boundary function to a closed ball in $R^{n}$ is continuous, the Fejér means converge to it uniformly on the closed ball, not merely on subballs of smaller radius.

2. Notation and preliminary results. Let $P$ denote the open upper half-plane in the complex z-plane, and $R$ (the real axis) its boundary. By $P^{n}$ we denote the Cartesian product of $n$ copies of $P$, and $R^{n}$ is then the distinguished boundary of $P^{n}$. We use usual vector nota-

AMS 1969 subject classifications. Primary 3040.

Key words and phrases. Bounded analytic functions, boundary values, Lindelöf theorem, Poisson integral, Tauberian theorem, Fejér means, polydisc. 
tions, denoting by $z=\left(z_{1}, \cdots, z_{n}\right)$ a point of $P^{n}, z_{j}=x_{j}+i y_{j}$, and by $x=\left(x_{1}, \cdots, x_{n}\right)$ a point of $R^{n} ; d x$ denotes Lebesgue measure in $R^{n}$, and $a, b$ positive scalars.

By $H^{\infty}\left(P^{n}\right)$ we denote the set of bounded holomorphic functions in $P^{n}$. To each $f \in H^{\infty}\left(P^{n}\right)$ is uniquely associated an element $\varphi$ of $L^{\infty}\left(R^{n}\right)$, its boundary function, by the Fatou relation

$$
\lim _{a \rightarrow 0} f\left(x_{1}+i a, \cdots, x_{n}+i a\right)=\varphi\left(x_{1}, \cdots, x_{n}\right) \text { a.e. }
$$

We can recover $f$ from $\varphi$ by the Poisson formula

$$
f(z)=\int \varphi(x-\xi) p(\xi, y) d \xi
$$

(here and elsewhere the integration is over $R^{n}$ if not specified otherwise) where

$$
p(x, y)=\pi^{-n} \prod_{j=1}^{n} y_{j}\left(x_{j}^{2}+y_{j}^{2}\right)^{-1}
$$

By $H^{\infty}\left(R^{n}\right)$ we denote the set of boundary functions of elements of $H^{\infty}\left(P^{n}\right)$.

For $h \in L^{1}\left(R^{n}\right)$, we write $h_{(a)}(x)=a^{-n} h\left(a^{-1} x\right)$. Finally, $B(x, a)$ denotes the closed ball in $R^{n}$ with center $x$ and radius $a$.

\section{Main results.}

THEOREM 1. Let $\varphi \in H^{\infty}\left(R^{n}\right), x^{0} \in R^{n}$, and suppose there exist $\lambda \in C, b>0$, and a function $\sigma(a)$ tending to zero as $a \rightarrow 0$, with the following properties. For each $a \leqq 1, B\left(x^{0}, a\right)$ contains a ball $K_{a}$ of radius ba such that ess sup $|\varphi(x)-\lambda|, x \in K_{a}$, does not exceed $\sigma(a)$. Then, for every $k \in L^{1}\left(R^{n}\right)$,

$$
\left(\varphi * k_{(a)}\right)\left(x^{0}\right)=\int \varphi\left(x^{0}-a x\right) k(x) d x \rightarrow \int k(x) d x
$$

as $a \rightarrow 0$.

REMARK 3.1. Clearly, if $\varphi(x) \rightarrow \lambda$ as $x \rightarrow x^{0}$ within an open cone with vertex at $x^{0}$ the hypothesis is satisfied.

CoROLLARY 3.2. Under the hypotheses of Theorem 1 , if $K$ is any open cone with vertex at $x^{0}$, the mean value of $\varphi$ over $K \cap B\left(x^{0}, a\right)$ tends to $\lambda$ as $a \rightarrow 0$.

Proof. Without loss of generality suppose $x^{0}$ is the origin. Choosing $k$ so that $k(-x)$ is the characteristic function of $K \cap B\left(x^{0}, 1\right)$ gives the result. 
CoROLlaRY 3.3. Under the hypotheses of Theorem 1, if $\varphi$ is the boundary function of $f \in H^{\infty}\left(P^{n}\right)$, then $\lim _{a \rightarrow 0} f\left(x_{1}^{0}+i a, \cdots, x_{n}^{0}+i a\right)=\lambda$.

PROOF. In view of (2.1) and (2.2),

$$
f\left(x_{1}^{0}+i a, \cdots, x_{n}^{0}+i a\right)=\left(\varphi * q_{(a)}\right)\left(x^{0}\right),
$$

where $q(x)=\pi^{-1}\left(1+x_{1}^{2}\right)^{-1} \cdots\left(1+x_{n}^{2}\right)^{-1}$. Hence, choosing $q$ for $k$ in (3.1) gives the result.

THEOREM 2. Let $G$ denote a subset of $L^{1}\left(R^{n}\right)$ such that no nonnull $\psi \in H^{\infty}\left(R^{n}\right)$ satisfies

$$
\int \psi(a x) g(x) d x=0, \text { all } g \in G, a>0 .
$$

If $\varphi \in H^{\infty}\left(R^{n}\right)$ satisfies

$$
\lim _{a \rightarrow 0} \int \varphi(a x) g(x) d x=\lambda \int g(x) d x, \text { all } g \in G,
$$

then

$$
\lim _{a \rightarrow 0} \int \varphi(a x) h(x) d x=\lambda \int h(x) d x, \text { all } h \in L^{1}\left(R^{n}\right) .
$$

REMARK 3.4. We give three examples, for $n=1$, of $G$ for which the hypothesis is easily verified. In each example, $G$ consists of a single function $g$.

(i) $g$ is the characteristic function of $[0,1]$,

(ii) $g$ is the characteristic function of $[-1,1]$,

(iii) $g(x)=\left(1+x^{2}\right)^{-1}$.

Hence, if $\varphi \in H^{\infty}(R)$ satisfies

$$
\lim _{a \rightarrow 0} \int \varphi(a x) g(x) d x=\lambda \int g(x) d x
$$

for any one of these $g$, it satisfies (3.3) for every $g \in L^{1}(R)$. In particular, the choice of $g$ in (iii) yields

Corollary 3.5. If $f \in H^{\infty}(P)$ satisfies $\lim _{a \rightarrow 0} f(i a)=\lambda$, its boundary function $\varphi$ satisfies (3.3) for every $g \in L^{1}(R)$.

From this it is quite easy to deduce that $f(z) \rightarrow \lambda$ as $z \rightarrow 0$ in a Stolz angle (Lindelöf's theorem). Other specializations of $g$ lead to these typical results: ${ }^{1}$

${ }^{1}$ (Cf. next page, line 2.) 
Corollary 3.6. If $\varphi \in H^{\infty}(R)$ satisfies $\lim _{a \rightarrow 0} a^{-1} \int_{0}^{a} \varphi(x) d x=\lambda$, then $\lim _{a \rightarrow 0} a^{-1} \int_{0}^{a} \varphi(x) d x=\lambda$.

CorollaRy 3.7. If $\varphi \in H^{\infty}(R)$ satisfies $\lim _{a \rightarrow 0}(2 a)^{-1} \int_{-a}^{a} \varphi(x) d x=\lambda$, then $\lim _{a \rightarrow 0} a^{-1} \int_{0}^{a} \varphi(x) d x=\lambda$.

The last three corollaries can also be proved quite directly by means of Wiener's Tauberian theorem; our proofs are however simpler, and independent of Wiener's theorem.

Definition. A compact set $E \subset R^{n}$ is well-rounded if constants $b, \delta$ can be found such that for every $x \in E$ and $a \leqq b, E \cap B(x, a)$ contains a ball of radius $\delta a$.

EXAMPLES. A closed interval in $R$ is well-rounded (we can take $\delta=\frac{1}{2}$ ); a polygonal region in $R^{2}$, or any region with piecewise smooth boundary and no cusps, is well-rounded.

THEOREM 3. Let $E$ be a well-rounded compact set in $R^{n}$. Suppose $\varphi$ is in $H^{\infty}\left(R^{n}\right)$ and coincides a.e. on $E$ with a continuous function $\varphi_{0}$. Then, if $k$ is any integrable function on $R^{n}$ satisfying $\int k d x=1,\left(\varphi * k_{(a)}\right)(x)$ converges to $\varphi_{0}(x)$, uniformly for $x \in E$, as $a \rightarrow 0$.

REMARK 3.8. If $F$ is bounded and holomorphic in the unit poly$\operatorname{disc} U^{n}$ (see Rudin [2]), and $\Phi$ its boundary function on the torus $T^{n}$, then $f\left(z_{1}, \cdots, z_{n}\right)=F\left(\exp \left[i z_{1}\right], \cdots, \exp \left[i z_{n}\right]\right)$ is in $H^{\infty}\left(P^{n}\right)$ and its boundary function is $\Phi\left(\exp \left[i x_{1}\right], \cdots, \exp \left[i x_{n}\right]\right)$. This enables us to apply the results of this paper to the polydisc-torus framework. In particular, Theorem 3 implies that quite general summability methods, applied to the Fourier series of $\Phi$, converge uniformly on well-rounded subsets of $T^{n}$ where $\Phi$ is continuous ("well-rounded" being suitably redefined for the torus). For example, taking $n=1$, $k(x)=\pi^{-1}(\sin x / x)^{2}$, we deduce that the Fejér means of the partial sums of the Fourier series of $\Phi \in H^{\infty}(T)$ converge uniformly to $\Phi$ on each closed interval to which the restriction of $\Phi$ is continuous.

4. Method of proof. Full details shall be given elsewhere; here we merely indicate the main ideas. The proofs are by "soft analysis", based upon two crucial properties of $H^{\infty}\left(R^{n}\right)$ :

(i) $H^{\infty}\left(R^{n}\right)$ is a weak ${ }^{*}$ closed subspace of $L^{\infty}\left(R^{n}\right)$.

(ii) Quasi-analyticity: a function in $H^{\infty}\left(R^{n}\right)$ which vanishes on a ball (or even on a set of positive measure) vanishes identically.

Let us write $\langle\varphi, g\rangle$ to denote $\int \varphi(x) g(-x) d x$, and define ${ }^{0} H^{\infty}\left(R^{n}\right)$ $=\left\{g \in L^{1}\left(R^{n}\right):\langle\varphi, g\rangle=0\right.$ for all $\left.\varphi \in H^{\infty}\left(R^{n}\right)\right\}$.

Lemma 4.1. Given $h \in L^{1}\left(R^{n}\right), \epsilon>0, b<1$, there is a constant $C(h, \epsilon, b)$ such that if $B$ is any ball of radius $b$ lying in the unit ball of $R^{n}$, there 
exists $g \in{ }^{0} H^{\infty}\left(R^{n}\right)$ such that $\|g\|_{1} \leqq C(h, \epsilon, b)$ and

$$
\int_{R^{n} \backslash B}|h(x)-g(x)| d x<\epsilon
$$

Proof (OUtline). For each $B$, the existence of $g \in{ }^{0} H^{\infty}\left(R^{n}\right)$ satisfying (4.1) follows by a standard duality argument from (i) and (ii). A compactness argument allows an estimate for $\|g\|_{1}$ independent of the particular choice of $B$.

Proof of Theorem 1. Without loss of generality, take $x^{0}=$ origin, $\lambda=0$. We have

$$
\int \varphi(a x) k(-x) d x=\int \varphi(a x)(k(-x)-g(x)) d x
$$

where $g=g_{a}$ is an element of ${ }^{0} H^{\infty}\left(R^{n}\right)$ that is at our disposal (here, in a nutshell, is the essence of our method).

By hypothesis, $B(0, a)$ contains a ball $K_{a}$ of radius $b a$ on which ess $\sup |\varphi(x)| \leqq \sigma(a)$, hence on the ball $J_{a}=a^{-1} K_{a}$, which is contained in the unit ball and has radius $b$, ess sup $|\varphi(a x)| \leqq \sigma(a)$. Now apply the lemma, with $h(x)=k(-x)$; we get, for a suitable $g=g_{a, e}$ in ${ }^{0} H^{\infty}\left(R^{n}\right)$

$$
\begin{aligned}
\left|\int \varphi(a x) k(-x) d x\right| & =\mid \int_{J_{a}} \varphi(a x)[k(-x)-g(x)] d x \\
& +\int_{R^{n} \backslash J_{a}} \varphi(a x)[k(-x)-g(x)] d x \mid \\
& \leqq \sigma(a)\left(\|k\|_{1}+C(k, \epsilon, b)\right)+\|\varphi\|_{\infty} \cdot \epsilon,
\end{aligned}
$$

hence

$$
\limsup _{a \rightarrow 0}\left|\int \varphi(a x) k(-x) d x\right| \leqq\|\varphi\|_{\infty} \cdot \epsilon
$$

and, $\epsilon$ being arbitrary, the lim sup is zero.

Proof of Theorem 2. We may suppose $\lambda=0$. Define

$$
M=\left\{h \in L^{1}\left(R^{n}\right): \lim _{a \rightarrow 0} \int \varphi(a x) h(x) d x=0\right\} .
$$

$M$ is a closed subspace of $L^{1}\left(R^{n}\right)$ containing all dilates of $g$ (i.e. the functions $x \rightarrow g(b x), b>0)$ for each $g \in G$. Also, ${ }^{0} H^{\infty}\left(R^{n}\right) \subset M$. Hence the theorem (i.e. $M=L^{1}\left(R^{n}\right)$ ) will be proved if ${ }^{0} H^{\infty}\left(R^{n}\right)$ together with the dilates of functions in $G$ span $L^{1}\left(R^{n}\right)$. By a duality argument, based on weak* closure of $H^{\infty}\left(R^{n}\right)$, this is ensured by the nonexistence of a nonnull solution $\psi \in H^{\infty}\left(R^{n}\right)$ to (3.2). 
The proof of Theorem 3 is along similar lines to that of Theorem 1, and shall not be given here.

5. Concluding remarks.

(a) It is natural to ask whether in Theorem $1 K_{a}$ may be required merely to be a set of measure $c a^{n}$, rather than a ball of this measure. To prove this by our method would require a corresponding strengthening of Lemma 4.1 whereby $B$ is allowed to be any set of measure $c$ lying in the unit ball. This we have only been able to carry out for $n=1$; for $n=1$ we can also prove an analogous extension of Theorem 3 , in terms of a weaker definition of "well-rounded" based on density.

(b) In the theorems of this paper, $H^{\infty}\left(R^{n}\right)$ can be replaced by $S(\wedge)$, where $\wedge$ is a closed subset of (the dual) $R^{n}$, defined as the set of $\varphi \in L^{\infty}\left(R^{n}\right)$ whose (distributional) Fourier transform is supported in $\wedge$, provided that $S(\wedge)$ has the quasi-analytic property.

(c) We do not know in general how to decide whether or not a given $G \subset L^{1}\left(R^{n}\right)$ satisfies the hypothesis of Theorem 2 . We wish also to call the readers' attention to the analogous problem, for which $G \subset L^{1}\left(R^{n}\right)$ is there no nonnull solution $\psi$ in $L^{\infty}\left(R^{n}\right)$ of (3.2)? This problem, the analog for dilates of the famous Wiener problem for translates arises naturally in studying boundary behaviour of harmonic functions. The case $n=1$ is reducible to the Wiener problem (cf. Gehring [1, pp. 107-110] for something similar), but the case $n>1$ seems to us fundamentally different in nature.

\section{REFERENCES}

1. F. W. Gehring, The Fatou theorem and its converse, Trans. Amer. Math. Soc. 85 (1957), 106-121.

2. Walter Rudin, Function theory on polydiscs, Benjamin, New York, 1969.

University of Michigan, Ann Arbor, Michigan 48104

AdDED IN PROOF October 31, 1970. Lee Rubel has kindly called our attention to the paper "Relations between bounded analy tic functions and their boundary functions" by T. K. Boehme, M. Rosenfeld, and Max L. Weiss, J. London Math. Soc. (2) 1 (1969), 609-618, where Corollaries 3.6 and 3.7 were obtained by a different method. 